\title{
Update Breast Cancer 2021 Part 4 - Prevention and Early Stages
}

\section{Update Mammakarzinom 2021 Teil 4 - Prävention und frühe Krankheitsstadien}

\section{(우(1) (오 $\ominus$}

\section{Authors}

Christoph Thomssen ${ }^{1}$, Tanja N. Fehm², Elmar Stickeler ${ }^{3}$, Peter A. Fasching ${ }^{4}$, Wolfgang Janni ${ }^{5}$, Cornelia Kolberg-Liedtke ${ }^{6,7,8}$, Hans-Christian Kolberg ${ }^{9}$, Diana Lüftner ${ }^{10}$, Volkmar Müller ${ }^{11}$, Florian Schütz ${ }^{12}$, Erik Belleville ${ }^{13}$, Simon Bader ${ }^{4}$, Michael Untch ${ }^{14}$, Manfred Welslau ${ }^{15}$, Marc Thill ${ }^{16}$, Andreas D. Hartkopf ${ }^{17}$, Hans Tesch ${ }^{18}$, Nina Ditsch ${ }^{19}$, Michael P. Lux ${ }^{20}$, Achim Wöckel ${ }^{21}$, Bahriye Aktas ${ }^{22}$, Andreas Schneeweiss ${ }^{23}$, Rachel Würstlein ${ }^{24}$

Affiliations

1 Department of Gynaecology, Martin-Luther-University

Halle-Wittenberg, Halle (Saale), Germany

2 Department of Gynecology and Obstetrics, University

Hospital Düsseldorf, Düsseldorf, Germany

3 Department of Gynecology and Obstetrics, RWTH University Hospital Aachen, Aachen, Germany

4 Erlangen University Hospital, Department of Gynecology and Obstetrics, Comprehensive Cancer Center ErlangenEMN, Friedrich-Alexander University Erlangen-Nuremberg, Erlangen, Germany

5 Department of Gynecology and Obstetrics, Ulm University Hospital, Ulm, Germany

6 Department of Gynecology and Obstetrics, University Hospital Essen, Essen, Germany

7 palleos healthcare, Wiesbaden, Germany

8 Phaon Scientific, Wiesbaden, Germany

9 Department of Gynecology and Obstetrics, Marienhospital Bottrop, Bottrop, Germany

10 Charité University Hospital, Department of Hematology, Oncology and Tumour Immunology, University Medicine Berlin, Berlin, Germany

11 Department of Gynecology, Hamburg-Eppendorf University Medical Center, Hamburg, Germany

12 Gynäkologie und Geburtshilfe, Diakonissen-StiftungsKrankenhaus Speyer, Speyer, Germany

13 ClinSol GmbH \& Co. KG, Würzburg, Germany

14 Clinic for Gynecology and Obstetrics, Breast Cancer Center, Genecologic Oncology Center, Helios Klinikum Berlin Buch, Berlin, Germany

15 Onkologie Aschaffenburg, Aschaffenburg, Germany

16 Agaplesion Markus Krankenhaus, Department of Gynecology and Gynecological Oncology, Frankfurt am Main, Germany

17 Department of Obstetrics and Gynecology, University of Tübingen, Tübingen, Germany
18 Oncology Practice at Bethanien Hospital, Frankfurt am Main, Germany

19 Department of Gynecology and Obstetrics, University Hospital Augsburg, Augsburg, Germany

20 Klinik für Gynäkologie und Geburtshilfe, Frauenklinik St. Louise, Paderborn, St. Josefs-Krankenhaus, Salzkotten, St. Vincenz Krankenhaus GmbH, Paderborn, Germany

21 Department of Gynecology and Obstetrics, University Hospital Würzburg, Würzburg, Germany

22 Klinik und Poliklinik für Gynäkologie, Universitätsklinikum Leipzig, Leipzig, Germany

23 National Center for Tumor Diseases (NCT), Heidelberg University Hospital and German Cancer Research Center, Heidelberg, Germany

24 Breast Center, Department of Gynecology and Obstetrics and CCC Munich LMU, LMU University Hospital, Munich, Germany

Key words

BRCA (breast cancer associated gene), breast, genetics, Her-2/neu (human epidermal growth factor receptor), hormonal receptor, breast cancer

Schlüsselwörter BRCA (breast cancer associated gene), Brust, Mamma, Genetik, Her-2/neu (humaner epidermaler Wachstumsfaktor-Rezeptor), Hormonrezeptor, Mammakarzinom

received

6. 12.2021

accepted after revision 
Bibliography

Geburtsh Frauenheilk 2022; 82: 206-214

DOI 10.1055/a-1724-9639

ISSN 0016-5751

(C) 2022. The Author(s).

This is an open access article published by Thieme under the terms of the Creative Commons Attribution-NonDerivative-NonCommercial-License, permitting copying and reproduction so long as the original work is given appropriate credit. Contents may not be used for commercial purposes, or adapted, remixed, transformed or built upon. (https://creativecommons.org/licenses/by-nc-nd/4.0/)

Georg Thieme Verlag KG, Rüdigerstraße 14,

70469 Stuttgart, Germany

Correspondence

Peter A. Fasching, MD

Erlangen University Hospital, Department of Gynecology and

Obstetrics, Comprehensive Cancer Center Erlangen EMN,

Friedrich Alexander University of Erlangen-Nuremberg

Universitätsstraße 21-23, 91054 Erlangen, Germany

peter.fasching@fau.de

$\Theta$

Deutsche Version unter:

https://doi.org/10.1055/a-1724-9639

\section{ABSTRACT}

This past year has seen new and effective options for further improving treatment outcome in many patients with earlystage breast cancer. Patients with hormone receptor-positive disease benefited significantly from the addition of the CDK4/ 6 inhibitor abemaciclib to endocrine adjuvant therapy. In triple-negative disease, data were presented for two treatment regimens. Patients with advanced disease (stage 2 and 3) benefit from neoadjuvant treatment with the immune checkpoint inhibitor pembrolizumab in combination with standard che- motherapy, regardless of PD-L1 expression. When neoadjuvant therapy has failed to achieve the desired remission in BRCA1 and BRCA2 mutations, the administration of the PARP inhibitor olaparib has demonstrated an impressive response. Other data address translational issues in HER2-positive breast cancer and neoadjuvant therapy approaches with the oral SERD giredestrant and the PARP inhibitor talazoparib. This review presents and analyses the findings of this year' $s$ most important study outcomes.

\section{ZUSAMMENFASSUNG}

Im vergangenen Jahr wurden für viele Patientinnen mit Mammakarzinom in frühem Krankheitsstadium neue und effektive Optionen für eine weitere Verbesserung der Behandlungsergebnisse gezeigt. Für Patientinnen mit hormonrezeptorpositiver Erkrankung zeigte sich ein signifikanter Zusatzeffekt durch den Einsatz des CDK4/6-Inhibitors Abemaciclib zusätzlich zur endokrinen adjuvanten Therapie. Bei triple-negativer Erkrankung wurden Daten für 2 Therapieprinzipien gezeigt. Patientinnen mit fortgeschrittener Erkrankung (Stadium 2 und 3) profitieren von dem neoadjuvanten Einsatz des Immuncheckpoint-Inhibitors Pembrolizumab unabhängig von der PD-L1-Expression in Kombination mit einer Standardchemotherapie. Bei BRCA1- oder BRCA2-Mutation wurde ein eindrucksvoller Benefit durch den Einsatz des PARP-Inhibitors Olaparib gezeigt, wenn die neoadjuvante Therapie nicht zur gewünschten Remission geführt hat. Weitere Daten betreffen translationale Fragestellungen beim HER2-positiven Mammakarzinom sowie neoadjuvante Therapieansätze mit dem oralen SERD Giredestrant und dem PARP-Inhibitor Talazoparib. In dieser Übersichtsarbeit werden die Ergebnisse der wichtigsten Studienergebnisse dieses Jahres vorgestellt und bewertet.

\section{Introduction}

In patient with early breast cancer, the gradual progress in adjuvant therapy has resulted in a significant improvement in the chances of cure. Important interventions such as dose-dense chemotherapy, pertuzumab and T-DM1 in HER2-positive cancer, $\mathrm{GnRH}$ agonists in premenopausal hormone receptor-positive disease, and carboplatin to optimise chemotherapy in triple-negative cancer have been incorporated in recent years. The focus is on new targeted drugs to further optimise adjuvant therapy and also on de-escalation of therapeutic measures through better identification of patients at risk, the reduction of medications and better supportive therapy. These were also prominent topics at recent congresses such as ASCO 2021 and ESMO 2021, which will be summarised in this review.

\section{Ten Breast Cancer Risk Genes You Should Know}

Since the 1990s, BRCA1 and BRCA2 genotyping has been part of counselling healthy women seeking consultation for hereditary breast and ovarian cancer. Following the large-scale trials published in early 2021, evidence is accumulating on which genes should be genotyped in panel testing. One commentary identified 10 genes for cancer-susceptibility [1]. BRCA1, BRCA2, PALB2, ATM and CHEK2 are clearly defined and validated breast cancer risk genes [2,3]. The data for TP53 also clearly indicate that it is a gene for breast cancer-susceptibility [1], but the low prevalence in these large-scale trials prevented researchers from calculating the corresponding risk [2,3]. Three other genes (BARD1, RAD51C and $R A D 51 D$ ) mainly increase the risk of hormone receptor-negative breast cancer [2,3]. Even if they do not significantly increase the lifetime risk because of this, hormone receptor-negative breast cancer is highly significant. The identification of mutation carriers is not only important for the prevention of breast cancer, but also to identify women at significantly increased risk of ovarian cancer. In terms of the risk genes stated, there is a large overlap wit BRCA1, BRCA2, PALB2, RAD51C and RAD51D [4]. In addition, 
BRIP1 should be genotyped, which, although not clearly associated with breast cancer risk [5], is useful in identifying women at high risk of ovarian cancer [4]. Thus, the following 10 genes could be regarded as a gene panel on which testing and counselling should focus.

- ATM

- BARD1

- bRCA1

- BRCA2

- BRIP1

- CHEK2

- PALB2

- RAD51C

- $R A D 51 D$

- TP53

The function of these genes is summarised in $>$ Table 1 . With the growing knowledge of which genes are validated risk factors in the development of breast cancer, potential attempts are also being made to apply this knowledge to the treatment of breast cancer. The effects of BRCA1/2 mutations in the neoadjuvant and adjuvant setting have already been reported in past studies. While chemotherapy in the neoadjuvant setting appears to improve efficacy [6-8], the reported effect on prognosis is inconsistent [9]. There are similar studies for other genes such as PALB2 or CHEK2 describing the effect on the prognosis of breast cancer patients, but no effects resulting in clinical implications [10 - 12]. However, mutations in PALB2 may be an indicator of response to olaparib, as most TNBC patients with a PALB2 mutation responded to olaparib therapy in a small study [13].

However, data from the metastatic setting have been lacking to date. PRAEGNANT, a recently published registry study, reported a mutation frequency of $5 \%$ for $B R C A 1 / 2$ mutations in patients with metastatic breast cancer [14]. Another about $5 \%$ of the patients carried a mutation in one of the other known breast cancer risk genes [14].

In this study, the difference in progression-free survival and overall survival between patients with and without a germ line mutation in one of the breast cancer risk genes was not statistically significant [14].

\section{Non-endocrine Based Treatment in Early Stages of the Disease}

Two important studies, the OlympiA study and the survival data from the KEYNOTE-522 study, have recently been published [15, $16]$. Both demonstrated clinically important advances in the treatment of patients with triple-negative breast cancer (TNBC), and the OlympiA trial also in the treatment of patients with HER2-negative, hormone receptor-positive breast cancer.

\section{Better event-free survival in TNBC patients with pembrolizumab statistically significant}

The KEYNOTE-522 study has already been published concerning one of its study objectives. In the KEYNOTE-522 trial, patients with stage 2 and 3 triple-negative breast cancer underwent neoadju-
- Table 1 Functional classification of the validated breast cancer risk genes.

\begin{tabular}{|l|l|l|l|}
\hline $\begin{array}{l}\text { Name } \\
\text { of gene }\end{array}$ & $\begin{array}{l}\text { involved in } \\
\text { homologous } \\
\text { recombination }\end{array}$ & $\begin{array}{l}\text { involved } \\
\text { in other } \\
\text { DNA repair } \\
\text { mechanisms }\end{array}$ & $\begin{array}{l}\text { validated } \\
\text { breast cancer } \\
\text { risk gene }\end{array}$ \\
\hline ATM & $\mathrm{X}$ & & $\mathrm{X}$ \\
\hline BARD1 & $\mathrm{X}$ & $\mathrm{X}$ \\
\hline BRCA1 & $\mathrm{X}$ & $\mathrm{X}$ & $\mathrm{X}$ \\
\hline BRCA2 & $\mathrm{X}$ & $\mathrm{X}$ & $\mathrm{X}$ \\
\hline BRIP1 & & & $\mathrm{X}$ \\
\hline CHEK2 & & & $\mathrm{X}$ \\
\hline PALB2 & $\mathrm{X}$ & & $\mathrm{X}$ \\
\hline RAD51C & $\mathrm{X}$ & $\mathrm{X}$ & $\mathrm{X}$ \\
\hline RAD51D & $\mathrm{X}$ & & \\
\hline TP53 & & & \\
\hline
\end{tabular}

vant treatment with standard chemotherapy including carboplatin and were compared with patients who also received supplemental pembrolizumab. Pembrolizumab treatment was continued in the pembrolizumab plus chemotherapy arm for six months following surgery [17]. In the final analysis of all 1174 patients participating in the survival analyses, the pCR rate was $55.6 \%$ in the chemotherapy arm and $63.0 \%$ in the pembrolizumab plus chemotherapy arm. Thus, the pembrolizumab plus chemotherapy arm benefited by $7.5 \%$ [18]. The fourth interim analysis presented robust data on the probability of event-free survival. With a median follow-up of 39.1 months, it was shown that the addition of pembrolizumab decreased the risk of relapse or death by $37 \%$ (hazard ratio: $0.63 ; 95 \% \mathrm{Cl}: 0.48-0.82$ ) [15]. This effect was statistically significant $(\mathrm{p}=0.00031)$. The probability of event-free survival at 36 months is $76.8 \%$ in the chemotherapy arm and $84.5 \%$ in the pembrolizumab plus chemotherapy arm. Interestingly enough, the relative benefit was present and similar in both patients with and without a PCR ( $\vee$ Fig. 1). Although there was no statistically significant difference in overall survival, probably due to the still short follow-up period, the numerical difference at 3 years was $2.8 \%$ (89.7\% in the pembrolizumab arm and $86.9 \%$ in the chemotherapy arm). The hazard ratio (HR) was 0.72 (95\% $\mathrm{Cl}$ : 0.51-1.02) [15]. More analyses with longer follow-up time are expected.

\section{Neoadjuvant data with durvalumab support the integration of PD-1/PD-L1 inhibitors into the treatment of early-stage disease}

Although the phase II GeparNuevo trial with 174 patients is much smaller than the KEYNOTE-522 trial, the results recently presented support the role of immuno-oncological regimens in patients with stage 2 and 3 TNBC [19]. Invasive disease-free survival (iDFS), distant disease-free survival (dDFS) and overall survival were secondary study objectives. The hazard ratios were $0.48(95 \% \mathrm{Cl}$ : 0.24-0.97; $p=0.0398$ ) for iDFS, 0.31 (95\% Cl: 0.13-0.74, $\mathrm{p}=0.0078)$ for dDFS and $0.24(95 \% \mathrm{Cl}: 0.08-0.72 ; \mathrm{p}=0.0108)$ 


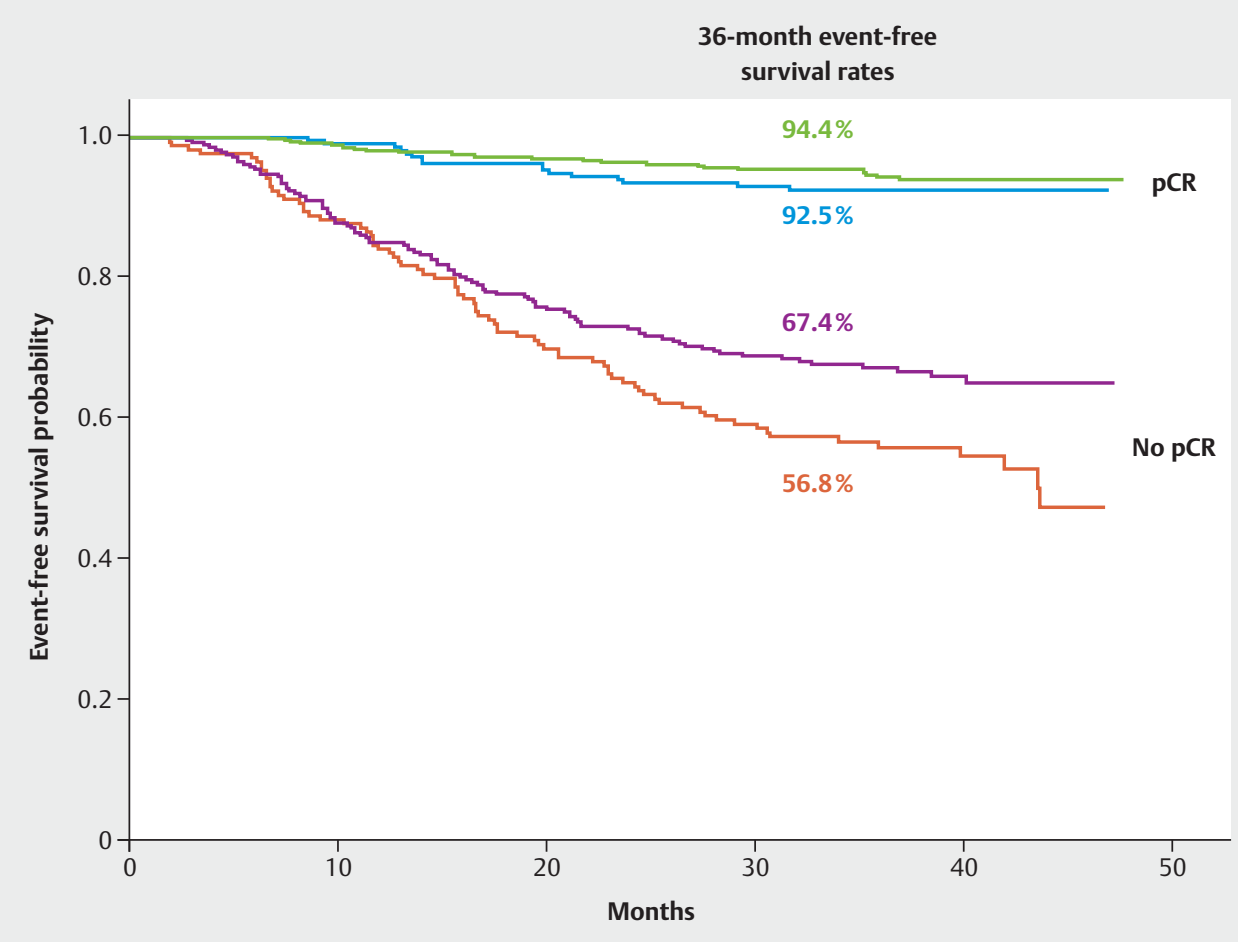

- Fig. 1 Event-free survival in the KEYNOTE-522 trial by randomisation arm and pCR group (data from [15]).

for overall survival. There was no evidence that this effect was limited to the group of patients with or without $\mathrm{PCR}$ or that the benefit of the checkpoint inhibitor was reflected in the attainment of pCR alone, even though the trial was not designed to draw such conclusions.

\section{Olaparib in adjuvant settings}

Since the PARP inhibitors olaparib and talazoparib have already been approved in the metastatic setting for HER2-negative patients with a BRCA1 or BRCA2 germ line mutation, the OlympiA trial for patients at high risk for relapse in the early stages of the disease has tested whether 12 months of adjuvant therapy with olaparib can improve iDFS. With a median follow-up of 2.5 years and 1836 patients randomised, iDFS was shown to improve with a HR of 0.58 ( $95 \% \mathrm{Cl}: 0.41-0.82$ ). The probability of disease-free survival (iDFS) at 36 months was $77.1 \%$ for the placebo arm and $85.9 \%$ for the olaparib arm [16]. Although the comparison showed no statistically significant difference in terms of overall survival, the mortality rate was reduced by $32 \%$ (HR: 0.68 ; $95 \%$ Cl: $0.44-1.05 ; p=0.02$ ) [16]. $18 \%$ of the enrolled patients had hormone receptor-positive breast cancer. While the treatment effect was numerically smaller in this group (HR: $0.70 ; 95 \% \mathrm{Cl}$ : 0.49-1.21) than in the TNBC cohort (HR: 0.52 ; $95 \% \mathrm{Cl}: 0.39-$ 0.69 ), no heterogeneity was demonstrated for the subgroups. One explanation might be the small number of cases.

Both the OlympiA trial and the KEYNOTE-522 trial are groundbreaking because they are changing the treatment landscape for patients with triple-negative breast cancer and HER2-negative, hormone receptor-positive breast cancer.

\section{Talazoparib in neoadjuvant settings}

After a small, neoadjuvant trial $(n=13)$ in patients with a $B R C A 1 / 2$ mutation achieved a median reduction in tumour volume of $88 \%$ with talazoparib monotherapy [20], the question arose as to how this therapy would fare in a larger group of patients. This question was tested in the NeoTALA trial [21]. This study analysed 48 HER2negative patients with a germ line BCRA1/2 mutation and treated with $0.75 \mathrm{mg}$ talazoparib per day as neoadjuvant monotherapy for 24 weeks. $45.8 \%$ of this population achieved a pCR. Especially against the backdrop of its markedly better tolerance compared to chemotherapy [22], this information is helpful to continue the development of corresponding therapeutic concepts.

\section{Role of carboplatin in the treatment of TNBC patients}

It was already known from the trials GeparSixto and CALBG 40603 that adding carboplatin to anthracycline- and taxane-based neoadjuvant chemotherapy increased PCR rates from $37-46 \%$ to $53-60 \%[23,24]$. In the GeparSixto trial, the addition of carboplatin to anthracycline- and taxane-based intensive neoadjuvant chemotherapy improved relapse-free survival in the TNBC population with a HR of 0.54 (95\% Cl: 0.34-0.93). Overall survival improved with a HR of 0.60 (95\% Cl: 0.32-1.12) [25]. In the CALGB 40603 trial, platinum-free chemotherapy did not differ in survival from platinum-based neoadjuvant chemotherapy [26]. Survival data from the BrighTNess study have now been published in this con- 
text [27]. In the BrighTNess study, patients were randomised into three treatment arms. Patients with early TNBC were enrolled regardless of their BRCA1/2 mutation status. The patients initially received either paclitaxel monotherapy, a combined regimen with paclitaxel and carboplatin or a regimen with paclitaxel, carboplatin and veliparib. All patients then received four cycles of doxorubicin and cyclophosphamide. The 4-year event-free survival of $78.2 \%$ in the paclitaxel-carboplatin-veliparib arm was comparable to the $79.3 \%$ survival in the paclitaxel-carboplatin arm. At $68.5 \%$, the paclitaxel-monotherapy arm was markedly lower [27]. Overall, the BrightNess trial therefore confirmed the efficacy of platinumbased chemotherapy in the neoadjuvant treatment of patients with triple-negative breast cancer. However, the interpretation of the data is complicated by the fact that the risk reduction obtained relative to the first event mainly concerned locoregional recurrence, less so distant metastasis.

\section{Treatment of Early-Stage HER2-positive Tumour Patients}

\section{De-escalating anti-HER2 therapy}

The duration of adjuvant anti-HER2 treatment with trastuzumab has been under discussion for some time. Treatment with trastuzumab in the (neo-)adjuvant setting over one year is well established by now [28-30]. The question of whether a two-year course of therapy would be better was clearly answered in the negative [31]. However, some other studies have been conducted comparing a one-year trastuzumab regimen with a shorter course of treatment [32-37]. The PERSEPHONE, HORG and PHARE trials compared one year of trastuzumab with a six-month course. The SOLD and Short-HER trials shortened adjuvant treatment even further, comparing a one-year course of trastuzumab with a nine-week regimen.

Recently, a meta-analysis of these data (12 months versus shorter therapy) in a non-inferiority design has been published. This analysis covered more than 11300 patients [38] and included three comparisons: all trials comparing 6 months with 12 months, and trials comparing 9 weeks with 12 months. The cut-off for noninferiority was seen at a hazard ratio of 1.19 to 1.25 , depending on which of the three analyses was performed. This corresponded to an absolute difference of $2 \%$ in invasive disease-free survival.

The meta-analysis confirmed the non-inferiority of 6 months versus 12 months. However, the non-inferiority of 9 weeks versus 12 months could not be confirmed [38]. Moreover, the quite extensive data on 12 months of trastuzumab in patients with earlystage disease raises doubts that adjuvant trastuzumab treatment for 6 months could become a new standard in the treatment of HER2-positive patients. The authors suggested that in the individual treatment setting - if necessary - it could be decided together with the patients whether treatment should be continued after 6 months, as the extension to 12 months only provided a marginal additional benefit of $0.7 \%$. Yet, it must also be taken into account that the treatment combination of the studies in the meta-analysis is rare nowadays. Many patients receive dual therapy (trastuzumab and pertuzumab) in the neoadjuvant setting, and in the ab- sence of complete remission may receive T-DM1 as postneoadjuvant treatment. Patients with trastuzumab mono often undergo de-escalation of chemotherapy with 12 cycles of paclitaxel according to the APT study. It is currently unclear whether in de-escalation of chemotherapy, further de-escalation may also be achieved by shortening the trastuzumab treatment period.

\section{De-escalation of chemotherapy}

ADAPT-HER2+HR is another trial addressing the de-escalation of anti-HER2 treatment [39]. This trial compared chemotherapyfree, neoadjuvant treatment with trastuzumab and pertuzumab $(T+P)$ versus treatment with paclitaxel, trastuzumab and pertuzumab $(T+P+P a c)$. Accordingly, both arms followed a de-escalating strategy compared to the standard treatment. The $T+P$ arm achieved a pCR rate (ypT0/is ypN0) of 34.4\% compared to a pCR rate of $90.5 \%$ in the $T+P+P a c$ arm [39]. Despite the markedly low PCR rate in the $T+P$ arm, it is of great interest which patients achieved a PCR without the addition of chemotherapy. For this reason, translational analyses have been performed despite the rather small number of cases, a total of 134 patients.

This allowed patients with highly HER2-expressing tumours, with non-basal-like tumours, with early treatment response, and patients with specific gene expression profiles to be identified as promising for a chemotherapy-free treatment regimen [39]. Nevertheless, these and other de-escalation studies are inadequate to replace the current standard.

\section{Endocrine-based Treatment in Early-Stage Disease}

\section{CDK4/6 inhibitors in adjuvant therapy}

After the monarchE trial with its rather short median follow-up of 15.5 months in terms of invasive disease-free survival (HR: 0.72; $95 \% \mathrm{Cl}$ : 0.56-0.92; favouring combined abemaciclib therapy) revealed positive data [40], the treatment was considered a possible option in patients with HER2-negative, hormone receptor-positive breast cancer, although the observation period was deemed too short to draw firm conclusions about the long-term value of the treatment. In San Antonio, data were published in 2020 with a longer median follow-up of 19.1 months (HR: 0.713 ; $95 \% \mathrm{Cl}$ : 0.583-0.871). Now another analysis with a median follow-up (FU) of 27.1 months has been presented [41]. In this analysis, the HR was 0.70 ( $95 \% \mathrm{Cl}: 0.59-0.82)$. The disease-free survival rate after 3 years was $83.4 \%$ in the standard endocrine treatment arm and $88.8 \%$ in the combination arm with abemaciclib. This corresponds to an absolute difference of $5.4 \%$ ( $\vee$ Fig. 2 ). Altogether, it can be stated that the relative improvement of the prognosis does not change much from analysis to analysis, which speaks for the stability of these outcomes. At the time of the 27-month followup analysis, $90 \%$ of the patients were already no longer on therapy, making it more likely that the difference between both randomisation arms would be maintained. In view of these current data, on 13 October 2021, the US Food and Drug Administration approved abemaciclib in the US for patients with HER2-negative, hormone receptor-positive breast cancer and positive lymph 


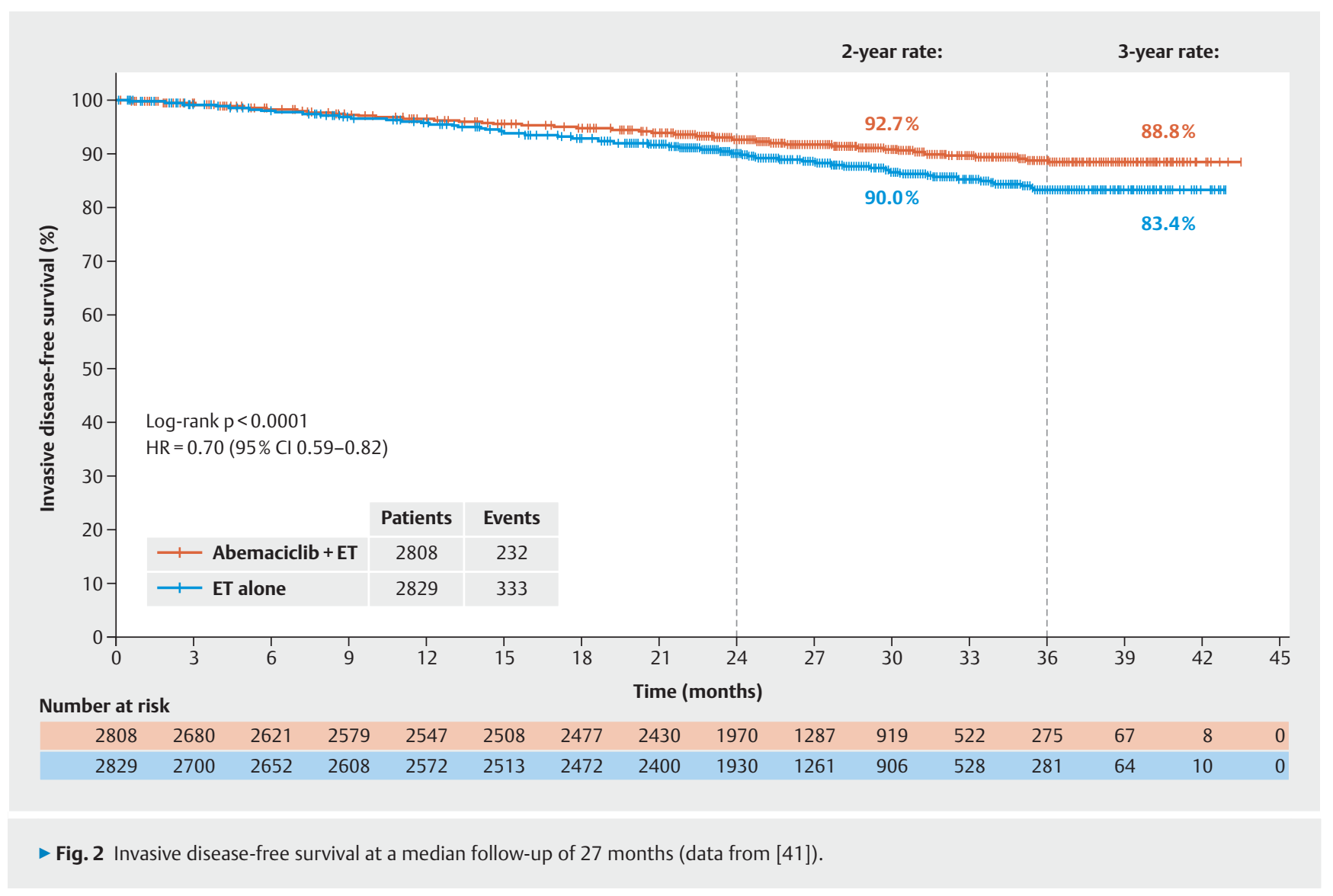

nodes and a Ki-67 $\geq 20 \%$ (as determined by the FDA approved test "MIB-1 pharmDx [Dako Omnis]"). It remains to be seen which criteria will be adopted by the European regulatory authority, as this definition does not cover the complete study population of the monarchE trial. Even though $\mathrm{Ki}-67$ is an excellent prognostic marker and can also predict response to chemotherapy, this marker has not yet been established in routine practice in all treatment centres [42-45].

In the wake of the negative outcomes of the PenelopeB and PALLAS trials with palbociclib, the monarchE trial with abemaciclib is the first adjuvant trial to demonstrate the adjuvant benefit of CDK4/6 inhibitor-based therapy. The NATALEE trial (TRIO033) is another study that has recruited 5000 patients and will answer the question of whether the addition of the third CDK4/6 inhibitor ribociclib in the adjuvant setting will have a positive impact on disease-free survival.

\section{Selective estrogen receptor degraders (SERD) in early-stage disease}

Initial clinical experience with SERDs was gained in trials for advanced disease [46-51]. For a general introduction to the topic of “oral SERDs”, see Lüftner D, Schütz F, Stickeler E et al. Update Breast Cancer 2021 Part 5 - Advanced Breast Cancer. Geburtshilfe Frauenheilkd 2022; 82: 215-225. doi:10.1055/a-1724-9569.
Recently, data from an interim analysis of the neoadjuvant coopERA trial have been presented. The coopERA trial compared 14-day neoadjuvant induction treatment with the peroral SERD giredestrant versus anastrozole in terms of reduction in proliferation as measured by Ki-67. After these initial 14 days, the respective anti-hormonal therapy was continued together with palbociclib for 16 weeks. In the cohort treated with giredestrant, the proliferation factor $\mathrm{Ki}-67$ was reduced by $80 \%$ versus $67 \%$ in the cohort treated with anastrozole. With a p-value of 0.0222 , the trial did not achieve the $p$-value of 0.01029 needed for formal statistical significance. Nevertheless, these outcomes are promising and support the rationale of studying this substance in other therapeutic settings. The lidERA trial, which compares adjuvant 5-year treatment with giredestrant versus guideline-based 5-year adjuvant endocrine treatment as chosen by the physician [52], is currently being initiated internationally. The AMEERA- 6 trial of the oral SERD amcenestrant, which is being tested in an adjuvant setting in a study population that has prematurely completed adjuvant endocrine therapy and is therefore at high risk of relapse, will start soon [53].

The field of oral SERDs has seen some new developments, such as the use of PROTAC (Proteolysis Targeting Chimera) technology [54]. These hetero-bifunctional molecules bind a ligand for a protein of interest (in this case the oestrogen receptor) on one side and the E3 ubiquitin ligase complex on the other. This triggers oestrogen receptor breakdown. A neoadjuvant study is now also being planned with this substance [55]. 


\section{Outlook}

The challenges lie in optimising treatment in high-risk diagnoses such as triple-negative breast cancer, which relapses early despite all measures to the contrary, and luminal cancer which relapse even late in the course of treatment. Developments, such as immune checkpoint inhibitors and PARP inhibitors, open up new and effective treatment options in biologically defined subgroups. For luminal cancer, continued development of endocrine-based therapy with oral selective estrogen receptor degrading substances (oral SERDs) and CDK4/6 inhibitors promises higher cure rates. On the other hand, we know that the improved prognosis in early breast cancer has come at the cost of massive overtreatment. Therefore, it is also important to de-escalate where possible. Possible approaches include the development of less neurotoxic taxanes, the avoidance of anthracyclines in many indications, antibody treatment alone with the avoidance of chemotherapy in some HER2-positive cancers, toxicity reduction through the use of antibody-substance conjugates in HER2-positive and triplenegative cancers, and also the identification of patients with luminal cancers (ER-pos./HER2-neg.) who can be adequately treated with adjuvant endocrine therapy alone.

Early-stage breast cancer still remains a challenge. The heterogeneity of this disease requires personalised treatment concepts with escalation where necessary and de-escalation where possible.

\section{Acknowledgements}

This paper evolved in part as a result of company funding from onkowissen.de, Hexal, Pfizer, Lilly, MSD, Gilead, and Novartis. No company had any part in the preparation and recommendations of this manuscript. Sole responsibility for the content of the manuscript rests with the authors.

\section{Conflict of Interest}

B. A. received honoria and travel grants from AstraZeneca, Gilead, Genomic Health, Roche, Novartis, Celgene, Lilly, MSD, Eisai, Teva, Tesaro, Daiichi Sankyo and Pfizer.

E. B. received honoraria from Novartis, Hexal, BMS, Lilly, Pfizer, Roche, MSD, BBraun and onkowissen.de for consulting, clinical research management or medical education activities.

S. B. has no conflict of interest.

N.D. has received honoraria from MSD, Roche, AstraZeneca, Teva, Pfizer, Novartis, Seagen, Gilead, MCI Healthcare.

P. A. F. reports personal fees from Novartis, grants from Biontech, personal fees from Pfizer, personal fees from Daiichi Sankyo, personal fees from AstraZeneca, personal fees from Eisai, personal fees from Merck Sharp \& Dohme, grants from Cepheid, personal fees from Lilly, personal fees from Pierre Fabre, personal fees from SeaGen, personal fees from Roche, personal fees from Hexal, personal fees from Agendia, personal fees from Gilead.

T. N. F. has participated on advisory boards for Amgen, Daiichi Sankyo, Novartis, Pfizer, and Roche and has received honoraria for lectures from Amgen, Celgene, Daiichi Sankyo, Roche, Novartis and Pfizer.

A.D.H. received speaker and consultancy honoraria from AstraZeneca, Genomic Health, Roche, Novartis, Celgene, Lilly, MSD, Eisai, Teva, Tesaro, Daiichi Sankyo, Hexal and Pfizer.

W.J. has received research Grants and/or honoraria from Sanofi-Aventis, Daiichi Sankyo, Novartis, Roche, Pfizer, Lilly, AstraZeneca, Chugai, GSK, Eisai, Cellgene and Johnson \& Johnson.
C. K.-L. has received honoraria from Roche, AstraZeneca, Celgene, Novartis, Pfizer, Lilly, Hexal, Amgen, Eisai, and SonoScape, honoraria for consultancy from Phaon Scientific, Novartis, Pfizer, and Celgene, research funding from Roche, Novartis, and Pfizer, and travel grants from Novartis and Roche.

H.-C. K. has received honoraria from Pfizer, Novartis, Roche, Genomic Health/Exact Sciences, Amgen, AstraZeneca, Riemser, Carl Zeiss Meditec, Teva, Theraclion, Janssen-Cilag, GSK, LIV Pharma, Lily, SurgVision, Onkowissen and MSD, travel support from Carl Zeiss Meditec, LIV Pharma, Novartis, Amgen, Pfizer, Daiichi Sankyo, Tesaro and owns stock of Theraclion SA and Phaon Scientific GmbH.

D. L. received honoraria from Amgen, AstraZeneca, Eli Lilly, GSK, Loreal, MSD, Novartis, Pfizer, Teva.

M.P. L. has participated on advisory boards for AstraZeneca, Lilly, MSD, Novartis, Pfizer, Eisai, Gilead, Exact Sciences, Pierre Fabre, Grünenthal, Daiichi Sankyo, PharmaMar and Roche and has received honoraria for lectures from MSD, Lilly, Roche, Novartis, Pfizer, Exact Sciences, Daiichi Sankyo, Grünenthal, Gilead, AstraZeneca, and Eisai. He is editorial board member of medactuell from medac.

V. M. received speaker honoraria from Amgen, AstraZeneca, Daiichi Sankyo, Eisai, Pfizer, MSD, Novartis, Roche, Teva, Seattle Genetics and consultancy honoraria from Genomic Health, Hexal, Roche, Pierre Fabre, Amgen, ClinSol, Novartis, MSD, Daiichi Sankyo, Eisai, Lilly, Tesaro, Seattle Genetics and Nektar. Institutional research support from Novartis, Roche, Seattle Genetics, Genentech. Travel grants: Roche, Pfizer, Daiichi Sankyo.

E. S. received honoraria from Roche, Celgene, AstraZeneca, Novartis, Pfizer, Tesaro, Aurikamed GmbH, MCI Deutschland GmbH, bsh medical communications $\mathrm{GmbH}$, Onkowissen TV.

A.S. received research grants from Celgene, Roche, honoraria from Amgen, AstraZeneca, Aurikamed, Bayer, Celgene, Clinsol, Connectmedica, Gilead, GSK, I-MED, Lilly, MCI Deutschland, Metaplan, MSD, Nanostring, Novartis, Onkowissen.de, Promedicis, Pfizer, Pierre Fabre, Roche, Seagen, Streamedup, Teva, Tesaro, Thieme and travel support from Celgene, Pfizer, Roche.

F. S. participated on advisory boards for Novartis, Lilly, Amgen and Roche and received honoraria for lectures from Roche, AstraZeneca, MSD, Novartis and Pfizer.

H. T. received honoraria from Novartis, Roche, Celgene, Teva, Pfizer, AstraZeneca and travel support from Roche, Celgene and Pfizer. C. T. received honoraria for advisory boards and lectures from Amgen, AstraZeneca, Celgene, Daiichi Sankyo, Eisai, Gilead, Lilly, MSD, Mylan, Nanostring, Novartis, Pfizer, Pierre Fabre, Puma, Roche, Seagen, Vifor. M. T. has participated on advisory boards for AstraZeneca, Clovis, Daiichi Sankyo, Eisai, Gilead Science, GSK, Lilly, MSD, Novartis, Organon, Pfizer, Exact Sciences, Pierre-Fabre, Seagen and Roche and has received honoraria for lectures from Clovis, Daiichi Sankyo, Eisai, GSK, Lilly, MSD, Roche, Novartis, Organon, Pfizer, Seagen, Exact Sciences, Viatris, and AstraZeneca and has received trial funding by Exact Sciences and Endomag. Manuscript support was done by Amgen, Celgene, ClearCut, pfm medical, Roche, Servier.

M.U. all honoraria went to the institution/employer: Abbvie, Amgen, AstraZeneca, Celgene, Daiichi Sankyo, Eisai, Lilly, MSD Merck, Mundipharma, Myriad Genetics, Pfizer, PUMA Biotechnology, Roche, Sanofi Aventis, Novartis, Pierre Fabre.

M.W. has participated on advisory boards for AstraZeneca, Lilly, MSD, Novartis, Pfizer and Roche.

A.W. participated on advisory boards for Novartis, Lilly, Amgen, Pfizer, Roche, Tesaro, Eisai and received honoraria for lectures from Novartis, Pfizer, Aurikamed, Roche, Celgene.

R.W. has received personal fees/travel support from Agendia, Amgen, Aristo, AstraZeneca, Boeringer Ingelheim, Carl Zeiss, Celgene, Clinsol, Daiichi Sankyo, Eisai, Exact Sciences, Genomic Health, GlaxoSmithKline, Hexal, Lilly, Medstrom Medical, MSD, Mundipharma, Nanostring, Novartis, Odonate, Paxman, Palleos, Pfizer, Pierre Fabre, PumaBiotechnology, Riemser, Roche, Sandoz/Hexal, Seattle Genetics, Tesaro Bio, Teva, Veracyte and Viatris. 


\section{References}

[1] Foulkes WD. The ten genes for breast (and ovarian) cancer susceptibility. Nat Rev Clin Oncol 2021; 18: 259-260

[2] Hu C, Hart SN, Gnanaolivu R et al. A Population-Based Study of Genes Previously Implicated in Breast Cancer. N Engl J Med 2021; 384: 440-451

[3] Breast Cancer Association Consortium; Dorling L, Carvalho S, Allen J et al. Breast Cancer Risk Genes - Association Analysis in More than 113,000 Women. N Engl J Med 2021; 384: 428-439

[4] Ramus SJ, Song H, Dicks E et al. Germline Mutations in the BRIP1, BARD1, PALB2, and NBN Genes in Women With Ovarian Cancer. J Natl Cancer Inst 2015; 107: djv214

[5] Easton DF, Lesueur F, Decker B et al. No evidence that protein truncating variants in BRIP1 are associated with breast cancer risk: implications for gene panel testing. J Med Genet 2016; 53: 298-309

[6] Hahnen E, Lederer B, Hauke J et al. Germline Mutation Status, Pathological Complete Response, and Disease-Free Survival in Triple-Negative Breast Cancer: Secondary Analysis of the GeparSixto Randomized Clinical Trial. JAMA Oncol 2017; 3: 1378-1385

[7] Wunderle M, Gass P, Häberle L et al. BRCA mutations and their influence on pathological complete response and prognosis in a clinical cohort of neoadjuvantly treated breast cancer patients. Breast Cancer Res Treat 2018; 171: 85-94

[8] Fasching PA, Loibl S, Hu C et al. BRCA1/2 Mutations and Bevacizumab in the Neoadjuvant Treatment of Breast Cancer: Response and Prognosis Results in Patients With Triple-Negative Breast Cancer From the GeparQuinto Study. J Clin Oncol 2018; 36: 2281-2287

[9] Copson ER, Maishman TC, Tapper WJ et al. Germline BRCA mutation and outcome in young-onset breast cancer (POSH): a prospective cohort study. Lancet Oncol 2018; 19: 169-180. doi:10.1016/S1470-2045 (17)30891-4

[10] Schmidt MK, Hogervorst F, van Hien R et al. Age- and Tumor SubtypeSpecific Breast Cancer Risk Estimates for CHEK2*1100delC Carriers. J Clin Oncol 2016; 34: 2750-2760

[11] Weischer M, Nordestgaard BG, Pharoah P et al. CHEK2*1100delC heterozygosity in women with breast cancer associated with early death, breast cancer-specific death, and increased risk of a second breast cancer. J Clin Oncol 2012; 30: 4308-4316

[12] Cybulski C, Kluźniak W, Huzarski T et al. Clinical outcomes in women with breast cancer and a PALB2 mutation: a prospective cohort analysis. Lancet Oncol 2015; 16: 638-644

[13] Tung NM, Robson ME, Ventz S et al. TBCRC048: Phase II Study of Olaparib for Metastatic Breast Cancer and Mutations in Homologous Recombination-Related Genes. J Clin Oncol 2020; 38: 4274-4282

[14] Fasching PA, Yadav S, Hu C et al. Mutations in BRCA1/2 and Other Panel Genes in Patients With Metastatic Breast Cancer-Association With Patient and Disease Characteristics and Effect on Prognosis. J Clin Oncol 2021; 39: 1619-1630

[15] Schmid P, Cortes J, Dent R et al. KEYNOTE-522: Phase III study of neoadjuvant pembrolizumab + chemotherapy vs. placebo + chemotherapy, followed by adjuvant pembrolizumab vs. placebo for early-stage TNBC. Ann Oncol 2021; 32: 1198-1200

[16] Tutt ANJ, Garber JE, Kaufman B et al. Adjuvant Olaparib for Patients with BRCA1- or BRCA2-Mutated Breast Cancer. N Engl J Med 2021; 384: 2394-2405

[17] Schmid P, Cortes J, Pusztai L et al. Pembrolizumab for Early Triple-Negative Breast Cancer. N Engl J Med 2020; 382: 810-821

[18] Merck Sharp \& Dohme Corp., FDA. Pembrolizumab: Combined FDA and Applicant ODAC Briefing Document for the Oncologic Drugs Advisory Committee (ODAC) Meeting on February 9, 2021. Accessed February 20, 2021 at: https://www.fda.gov/media/145654/download
[19] Loibl S, Schneeweiss A, Huober JB et al. Durvalumab improves long-term outcome in TNBC: results from the phase II randomized GeparNUEVO study investigating neodjuvant durvalumab in addition to an anthracycline/taxane based neoadjuvant chemotherapy in early triple-negative breast cancer (TNBC). J Clin Oncol 2021; 39 (15_suppl): 506

[20] Litton JK, Scoggins M, Ramirez DL et al. A feasibility study of neoadjuvant talazoparib for operable breast cancer patients with a germline BRCA mutation demonstrates marked activity. NPJ Breast Cancer 2017; 3: 49

[21] Litton JK, Beck JT, Jones JM et al. Neoadjuvant talazoparib in patients with germline BRCA1/2 (gBRCA1/2) mutation-positive, early HER2-negative breast cancer (BC): Results of a phase 2 study. J Clin Oncol 2021; 39 (15_suppl): 505

[22] Ettl J, Quek RGW, Lee KH et al. Quality of life with talazoparib versus physician's choice of chemotherapy in patients with advanced breast cancer and germline BRCA1/2 mutation: patient-reported outcomes from the EMBRACA phase III trial. Ann Oncol 2018; 29: 1939-1947

[23] von Minckwitz G, Schneeweiss A, Loibl S et al. Neoadjuvant carboplatin in patients with triple-negative and HER2-positive early breast cancer (GeparSixto; GBG 66): a randomised phase 2 trial. Lancet Oncol 2014; 15: 747-756

[24] Sikov WM, Berry DA, Perou CM et al. Impact of the addition of carboplatin and/or bevacizumab to neoadjuvant once-per-week paclitaxel followed by dose-dense doxorubicin and cyclophosphamide on pathologic complete response rates in stage II to III triple-negative breast cancer: CALGB 40603 (Alliance). J Clin Oncol 2015; 33: 13-21

[25] Loibl S, Weber KE, Timms KM et al. Survival analysis of carboplatin added to an anthracycline/taxane-based neoadjuvant chemotherapy and HRD score as predictor of response - final results from GeparSixto. Ann Oncol 2018; 29: 2341-2347

[26] Sikov WM, Polley MY, Twohy E et al. CALGB (Alliance) 40603: Long-term outcomes (LTOs) after neoadjuvant chemotherapy (NACT) \pm carboplatin (Cb) and bevacizumab (Bev) in triple-negative breast cancer (TNBC). J Clin Oncol 2019; 37 (15_suppl): 591

[27] Loibl S, Sikov W, Huober ] et al. Event-free survival (EFS), overall survival $(\mathrm{OS})$, and safety of adding veliparib (V) plus carboplatin (Cb) or carboplatin alone to neoadjuvant chemotherapy in triple-negative breast cancer (TNBC) after $\geq 4$ years of follow-up: BrighTNess, a randomized phase III trial. Ann Oncol 2021; 32 (suppl_5): S407-S446

[28] Slamon D, Eiermann W, Robert $\mathrm{N}$ et al. Adjuvant trastuzumab in HER2positive breast cancer. N Engl J Med 2011; 365: 1273-1283

[29] Perez EA, Romond EH, Suman V] et al. Four-year follow-up of trastuzumab plus adjuvant chemotherapy for operable human epidermal growth factor receptor 2-positive breast cancer: joint analysis of data from NCCTG N9831 and NSABP B-31. J Clin Oncol 2011; 29: 3366-3373

[30] Piccart-Gebhart M], Procter M, Leyland-Jones B et al. Trastuzumab after adjuvant chemotherapy in HER2-positive breast cancer. N Engl J Med 2005; 353: 1659-1672

[31] Goldhirsch A, Gelber RD, Piccart-Gebhart M] et al. 2 years versus 1 year of adjuvant trastuzumab for HER2-positive breast cancer (HERA): an open-label, randomised controlled trial. Lancet 2013; 382: 1021-1028

[32] Conte P, Frassoldati A, Bisagni $G$ et al. Nine weeks versus 1 year adjuvant trastuzumab in combination with chemotherapy: final results of the phase III randomized Short-HER studył. Ann Oncol 2018; 29: 23282333

[33] Joensuu H, Fraser J, Wildiers $\mathrm{H}$ et al. Effect of Adjuvant Trastuzumab for a Duration of 9 Weeks vs. 1 Year With Concomitant Chemotherapy for Early Human Epidermal Growth Factor Receptor 2-Positive Breast Cancer: The SOLD Randomized Clinical Trial. JAMA Oncol 2018; 4: 11991206

[34] Mavroudis D, Saloustros E, Malamos N et al. Six versus 12 months of adjuvant trastuzumab in combination with dose-dense chemotherapy for women with HER2-positive breast cancer: a multicenter randomized study by the Hellenic Oncology Research Group (HORG). Ann Oncol 2015; $26: 1333-1340$ 
[35] Mavroudis D, Saloustros E, Malamos N et al. Corrigendum to Six versus 12 months of adjuvant trastuzumab in combination with dose-dense chemotherapy for women with HER2-positive breast cancer: a multicenter randomized study by the Hellenic Oncology Research Group (HORG): Annals of Oncology, Volume 26, Issue 7, July 2015, Pages 1333-1340. Ann Oncol 2020; 31: 444-445

[36] Pivot X, Romieu G, Debled M et al. 6 months versus 12 months of adjuvant trastuzumab in early breast cancer (PHARE): final analysis of a multicentre, open-label, phase 3 randomised trial. Lancet 2019; 393: 25912598

[37] Earl HM, Hiller L, Vallier AL et al. PERSEPHONE: 6 versus 12 months ( $\mathrm{m}$ ) of adjuvant trastuzumab in patients (pts) with HER2 positive $(+)$ early breast cancer (EBC): Randomised phase 3 non-inferiority trial with definitive 4-year (yr) disease-free survival (DFS) results. J Clin Oncol 2018; 36 (15_suppl): 506

[38] Earl HM, Hiller L, Dunn JA et al. LBA11 - Individual patient data metaanalysis of 5 non-inferiority RCTs of reduced duration single agent adjuvant trastuzumab in the treatment of HER2 positive early breast cancer. Ann Oncol 2021; 32 (Suppl. 5): S1283

[39] Harbeck N, Gluz O, Christgen M et al. De-escalated neoadjuvant pertuzumab+trastuzumab with or without paclitaxel weekly in HR-/HER2+ early breast cancer: ADAPT-HR-/HER2+ biomarker and survival results. J Clin Oncol 2021; 39 (15_suppl): 503

[40] Johnston SRD, Harbeck N, Hegg R et al. Abemaciclib Combined With Endocrine Therapy for the Adjuvant Treatment of HR+, HER2-, Node-Positive, High-Risk, Early Breast Cancer (monarchE). J Clin Oncol 2020; 38: 3987-3998

[41] Harbeck N, Rastogi P, Martin M et al. Adjuvant abemaciclib combined with endocrine therapy for high-risk early breast cancer: updated efficacy and Ki-67 analysis from the monarchE study. Ann Oncol 2021; 32: 1571-1581

[42] Yerushalmi R, Woods R, Ravdin PM et al. Ki67 in breast cancer: prognostic and predictive potential. Lancet Oncol 2010; 11: 174-183

[43] Fasching PA, Gass P, Häberle L et al. Prognostic effect of Ki-67 in common clinical subgroups of patients with HER2-negative, hormone receptor-positive early breast cancer. Breast Cancer Res Treat 2019; 175: 617625

[44] Fasching PA, Heusinger K, Haeberle L et al. Ki67, chemotherapy response, and prognosis in breast cancer patients receiving neoadjuvant treatment. BMC Cancer 2011; 11: 486

[45] Nielsen TO, Leung SCY, Rimm DL et al. Assessment of Ki67 in Breast Cancer: Updated Recommendations From the International Ki67 in Breast Cancer Working Group. J Natl Cancer Inst 2021; 113: 808-819

[46] Bardia A, Kaklamani V, Wilks S et al. Phase I Study of Elacestrant (RAD1901), a Novel Selective Estrogen Receptor Degrader, in ER-Positive, HER2-Negative Advanced Breast Cancer. J Clin Oncol 2021; 39 : $1360-1370$
[47] Lim E, Beeram M, Prawira A et al. EMBER: A phase 1a/b trial of LY3484356, a novel, oral selective estrogen-receptor degrader (SERD), in advanced ER plus breast cancer and endometroid endometrial cancer. Cancer Res 2021. doi:10.1158/1538-7445.Sabcs20-ot-09-03

[48] Linden HM, Campone M, Bardia A et al. Abstract PD8-08: A phase 1/2 study of SAR439859, an oral selective estrogen receptor (ER) degrader (SERD), as monotherapy and in combination with other anti-cancer therapies in postmenopausal women with ER-positive $(E R+) /$ human epidermal growth factor receptor 2-negative (HER2-) metastatic breast cancer (mBC): AMEERA-1. Proceedings of the 2020 San Antonio Breast Cancer Virtual Symposium, San Antonio, TX, USA. December 8-11, 2020. doi:10.1158/1538-7445.SABCS20-PD8-08

[49] Baird R, Oliveira M, Ciruelos Gil EM et al. Abstract PS11-05: Updated data from SERENA-1: A Phase 1 dose escalation and expansion study of the next generation oral SERD AZD9833 as a monotherapy and in combination with palbociclib, in women with ER-positive, HER2-negative advanced breast cancer. Cancer Res 2021; 81 (4 Supplement): PS11-05

[50] Jhaveri KL, Lim E, Hamilton EP et al. A first-in-human phase 1a/b trial of LY3484356, an oral selective estrogen receptor (ER) degrader (SERD) in $E R+$ advanced breast cancer $(\mathrm{aBC})$ and endometrial endometrioid cancer (EEC): Results from the EMBER study. J Clin Oncol 2021; 39 (15_suppl): 1050

[51] Hamilton EP, Wang JS, Pluard T et al. Abstract PD8-06: Phase I/II trial of H3B-6545, a novel selective estrogen receptor covalent antagonist (SER$\mathrm{CA})$, in estrogen receptor positive (ER+), human epidermal growth factor receptor 2 negative (HER2-) advanced breast cancer. Proceedings of the 2020 San Antonio Breast Cancer Virtual Symposium, San Antonio, TX, USA. December 8-11, 2020. doi:10.1158/1538-7445.SABCS20PD8-06

[52] ClinicalTrials.gov. NCT04961996, A Study Evaluating the Efficacy and Safety of Adjuvant Giredestrant Compared With Physician's Choice of Adjuvant Endocrine Monotherapy in Participants With Estrogen Receptor-Positive, HER2-Negative Early Breast Cancer (lidERA Breast Cancer). Accessed October 17, 2021 at: https://clinicaltrials.gov/ct2/show/ NCT04961996

[53] Sanofi Aventis Groupe. Sanofi partnering with leading academic cooperative groups to study amcenestrant in the adjuvant setting for patients with estrogen receptor positive breast cancer. Accessed October 16, 2021 at: https://www.sanofi.com/en/media-room/press-releases/2021/ 2021-06-04-07-00-00-2241809

[54] Snyder LB, Flanagan J], Qian Y et al. Abstract 44: The discovery of ARV-471, an orally bioavailable estrogen receptor degrading PROTAC for the treatment of patients with breast cancer. Cancer Res 2021; 81 (13 Supplement): 44

[55] Arvinas. Arvinas and Pfizer Announce Global Collaboration to Develop and Commercialize PROTAC ${ }^{\circledR}$ Protein Degrader ARV-471. Accessed October 16, 2021 at: https://ir.arvinas.com/news-releases/news-releasedetails/arvinas-and-pfizer-announce-global-collaboration-develop-and 